\title{
Implementation of a "see and treat" cervical cancer prevention program linked to HIV care in Zambia KS Pfaendler*1,2,3, MH Mwanahamuntu, ${ }^{1,4}$, VV Sahasrabuddhe ${ }^{5}$, JSA Stringer ${ }^{1,2}$, MI Hicks ${ }^{6}$ and GP Parham ${ }^{1,2,4}$
}

Address: ${ }^{1}$ Centre for Infectious Disease Research in Zambia, Lusaka, Zambia, ${ }^{2}$ University of Alabama at Birmingham, Birmingham, Alabama, USA, ${ }^{3}$ University of Pittsburgh School of Medicine, Pittsburgh, Pennsylvania, USA, ${ }^{4}$ University Teaching Hospital; Lusaka, Zambia, ${ }^{5}$ Vanderbilt University, Nashville, Tennessee, USA and ${ }^{6}$ Michigan Cancer Institute, Detroit, Michigan, USA

* Corresponding author

from II th International Conference on Malignancies in AIDS and Other Acquired Immunodeficiencies (ICMAOI): Basic, Epidemiologic, and Clinical Research

Bethesda, MD, USA. 6-7 October 2008

Published: 17 June 2009

Infectious Agents and Cancer 2009, 4(Suppl 2):O2I doi:I0.I 186/I750-9378-4-S2-O2 I

This abstract is available from: http://www.infectagentscancer.com/content/4/S2/O2I

(c) 2009 Pfaendler et al; licensee BioMed Central Ltd.

\section{Objective}

To establish a public-sector "see and treat" cervical cancer prevention program in Zambia by linking services to an HIV care and treatment infrastructure.

\section{Methods}

We modeled our infrastructure after a successful PEPFARfunded HIV care and treatment program and selected HIVinfected women as our initial target population. Zambian nurses underwent classroom and clinically-mentored training to become primary service providers for screening women for cervical lesions using visual inspection with acetic acid (VIA) and treatment with cryotherapy, when indicated. Women with cryotherapy-ineligible lesions were referred to the university hospital where physicians were trained to perform punch biopsy and loop electrosurgical excision procedure (LEEP) for histologic evaluation. We utilized telecervicography for distance consultation as well as reviewing digital images weekly for quality assurance and continuing education. Patients with invasive cancer were referred for hysterectomy, radiation or palliation, depending on the stage of their disease

\section{Results}

Between January 2006 and October 2007 we established 14 prevention sites in outlying government-operated public health clinics and a modern outpatient evaluation center. During this 22-month period 8,823 women were screened, 41.5 percent of whom were HIV-infected. The 15 specially-trained nurses independently managed 83.3 percent of clients in the outlying clinics and referred the remaining 16.7 percent for further evaluation. Four physicians managed the outpatient evaluation center, performing punch biopsy or LEEP, the latter with minimal intraand post-operative complications. Pathologic analysis confirmed 144 high-grade lesions (CIN2/3) and 149 invasive cancers ( $58 \%$ micro invasive).

\section{Conclusion}

We successfully established the first phase of a population-based "see and treat" cervical cancer prevention program in Zambia by linking the services to an HIV care and treatment program, integrating them into governmentoperated public health clinics and utilizing task-shifting and distance consultation to optimize care provision. 